\title{
NAKAYAMA AUTOMORPHISM OF A CLASS OF GRADED ALGEBRAS
}

\author{
J.-F. LÜ, X.-F. MAO, AND J.J. ZHANG
}

\begin{abstract}
Aвstract. The Nakayama automorphism of a class of connected graded Artin-Schelter regular algebras is calculated explicitly.
\end{abstract}

\section{INTRODUCTION}

Throughout let $\mathbb{k}$ denote a base field and assume that char $\mathbb{k} \neq 2$. All vector spaces and algebras are over $\mathbb{k}$.

We start with the definition of a class of algebras, denoted by $A(Q, C, s)$, as follows:

Let $Q:=\left(q_{i j}\right)_{n \times n}$ and $C:=\left(c_{i j}\right)_{n \times n}$ be two $n \times n$-matrices in $M_{n}(\mathbb{k})$ such that

$$
q_{i i}=1, \quad q_{j i}=q_{i j}^{-1}, \quad \text { for all } i \text { and } j,
$$

and

$$
c_{i i}=0, \quad c_{j i}=-q_{i j}^{-1} c_{i j}=-q_{j i} c_{i j}, \quad \text { for all } i \text { and } j .
$$

We may call $C$ a skew $Q$-symmetric matrix. Let $s$ be an integer such that $1 \leq s \leq n$ and let $\Omega$ denote $\sum_{i=1}^{s} t_{i}^{2}$, considered as an element in the free algebra $\mathbb{k}\left\langle t_{1}, t_{2}, \cdots, t_{n}\right\rangle$. Define

$$
A(Q, C, s)=\mathbb{K}\left\langle t_{1}, \cdots, t_{n}\right\rangle /\left(t_{j} t_{i}-q_{i j} t_{i} t_{j}-c_{i j} \Omega, \forall i, j\right) .
$$

If $c_{i j}=0$ for all $i, j$, it becomes the usually skew polynomial ring $\mathbb{k}_{q_{i j}}\left[t_{1}, \cdots, t_{n}\right]$. So we will only consider the case when some of $c_{i j}$ s are nonzero. By carefully choosing the parameters $Q$ and $C$, we can obtain plenty of interesting algebras, though all of them are Ore extensions (at least when $s<n$, see Proposition 1.6). Some examples are given in Section 2 . The algebra $A(Q, C, s)$ can also be viewed as a deformation of $\mathbb{k}_{q_{i j}}\left[t_{1}, \cdots, t_{n}\right]$ for some special values $\left(q_{i j}\right)_{n \times n}$.

By definition, $A(Q, C, s)$ is a quadratic algebra. The only result of this paper is to give some conditions on $Q$ and $C$ such that $A(Q, C, s)$ is Koszul, strongly noetherian, CohenMacaulay, Artin-Schelter regular, and Auslander regular of global dimension $n$ (and it is also a graded skew Clifford algebra); and when in this situation, we give an explicit formula of the Nakayama automorphism of the algebra $A(Q, C, s)$ in terms of a linear map associated to the basis $\left\{t_{1}, t_{2} \cdots, t_{n}\right\}$. We omit the definitions of some standard terms mentioned as above; these can be found in, for example, [ASZ, CV1, Le, RRZ1, RRZ2, SZ, ZZ]. However, we will recall the definition of the Nakayama automorphism in Definition 0.3 below because it is the objective of this paper.

2010 Mathematics Subject Classification. 16E65, 16W50, 16E10.

Key words and phrases. Nakayama automorphism, Artin-Schelter regular algebra. 
For simplicity, $A(Q, C, s)$ is sometimes denoted by $A(Q, C)$ or $A$. Consider the following conditions for $Q$ and $C$ :

$$
\begin{array}{rlrl}
q_{j \alpha}^{2} & =q_{j 1}^{2}, & & \text { for all } j \text { and all } \alpha=2, \cdots, s \\
q_{i \alpha} q_{j \alpha} c_{i j} & =q_{1 \alpha}^{2} c_{i j}, & \text { for all } \alpha \text { and all } i, j \neq \alpha \\
q_{i \alpha} c_{i \alpha} & =c_{i \alpha}, & & \text { for all } \alpha \leq s \text { and all } i \\
c_{\alpha j} c_{k l}-q_{j l} c_{\alpha l} c_{k j}+q_{l k} q_{j k} c_{\alpha k} c_{l j} & =0, & & \text { for all } \alpha \leq s \text { and all } j<l<k \\
c_{\alpha k} c_{l j}-q_{l k} c_{\alpha l} c_{k j}+q_{j l} q_{j k} c_{\alpha j} c_{k l} & =0, & & \text { for all } \alpha \leq s \text { and all } j<l<k \\
\operatorname{det}\left(I_{s \times s}-\left(c_{i j}\right)_{s \times s}\right) & \neq 0 . & &
\end{array}
$$

Remark 0.1. It follows from (E0.0.1), E0.0.2) and E0.0.5) that $C_{s}:=\left(c_{i j}\right)_{s \times s}$ is skew symmetric, though the bigger matrix $C:=\left(c_{i j}\right)_{n \times n}$ may not be, see Example 2.1 Hence E0.0.8) is equivalent to

$$
\operatorname{det}\left(I_{s \times s}+\left(c_{i j}\right)_{s \times s}\right) \neq 0 .
$$

Theorem 0.2. Assume char $\mathbb{k} \neq 2$. Suppose $Q$ and $C$ satisfy (E0.0.1)-(E0.0.8). Then

(1) $A(Q, C)$ is a Koszul Artin-Schelter regular algebra of global dimension $n$ with Hilbert series $\frac{1}{(1-t)^{n}}$.

(2) It is a strongly noetherian, Cohen-Macaulay, and Auslander regular domain.

(3) The Nakayama automorphism of $A(Q, C)$ is determined by

$$
\begin{aligned}
& \mu_{A(Q, C)}: t_{j}\left\{\begin{array}{lc}
\sum_{i=1}^{s} \sum_{l=1}^{s} a_{i l} c_{l j}\left(1+q_{j 1}^{2}\right)\left(\prod_{w=1}^{n} q_{w j}\right) t_{i}+\left(\prod_{w=1}^{n} q_{w j}\right) t_{j}, & \text { if } j>s \\
\sum_{i=1}^{s} b_{i j} q_{j 1}^{2}\left(\prod_{w=1}^{n} q_{w j}\right) t_{i}, & \text { if } j \leq s .
\end{array}\right. \\
& \text { where }\left(a_{i j}\right)_{s \times s}=\left[I_{s \times s}-\left(c_{i j}\right)_{s \times s}\right]^{-1} \text { and }\left(b_{i j}\right)_{s \times s}=\left[I_{s \times s}-\left(c_{i j}\right)_{s \times s}\right]^{-1}\left[I_{s \times s}+\left(c_{i j}\right)_{s \times s}\right] .
\end{aligned}
$$

As a consequence, $A(Q, C)$ is a graded skew Clifford algebra in the sense of CassidyVancliff [CV1, CV2], see Proposition 1.5 Assisted by any computing software, say Maple, after finding a solution to system E0.0.1 - E0.0.8) (which consists of quite naive equations), one can construct immediately an Artin-Schelter regular algebra of dimension $n$. This is similar to the ideas of Cassidy-Vancliff in [CV1, CV2].

As promised we recall the definition of the Nakayama automorphism, see [RRZ1] for more information.

Definition 0.3. Let $A$ be an algebra over $\mathbb{k}$. Let $A^{e}=A \otimes_{\mathbb{k}} A^{o p}$.

(1) $A$ is called skew Calabi-Yau if

(i) $A$ is homologically smooth, that is, $A$ has a projective resolution in the category $A^{e}$-Mod that has finite length and each term in the projective resolution is finitely generated, and

(ii) there is an integer $d$ and an algebra automorphism $\mu$ of $A$ such that

$$
\operatorname{Ext}_{A^{e}}^{i}\left(A, A^{e}\right) \cong \begin{cases}0 & i \neq d \\ { }^{1} A^{\mu} & i=d,\end{cases}
$$

as $A$-bimodules, where 1 denotes the identity map of $A$.

(2) If E0.3.1 holds for some algebra automorphism $\mu$ of $A$, then $\mu$ is called the Nakayama automorphism of $A$, and is usually denoted by $\mu_{A}$. Note that $\mu_{A}$ (if exists) is unique up to inner automorphisms of $A$.

(3) $A$ is called Calabi-Yau if it is skew Calabi-Yau and $\mu_{A}$ is inner, or equivalently, $\mu_{A}$ can be chosen to be the identity map after changing a bimodule generator of ${ }^{1} A^{\mu}$. 
When $A$ is a connected graded domain, every unit of $A$ is a nonzero scalar, which is central. In this case, every inner automorphism of $A$ is the identity map and the Nakayama automorphism of $A$ (if exists) is unique.

By [RRZ1, Lemma 1.2], a connected graded algebra is skew Calabi-Yau if and only if it is Artin-Schelter regular. The Nakayama automorphism of an Artin-Schelter regular algebra (or more generally a skew Calabi-Yau algebra) is an important invariant, which is closely related to the study of

(1) rigid dualizing complexes [V1] and the Calabi-Yau property [RRZ1, RRZ2];

(2) the Poincaré duality (or Van den Bergh duality) between Hochschild homology and Hochschild cohomology [V2];

(3) the automorphism group of Artin-Schelter regular algebras and group/Hopf algebra actions on Artin-Schelter regular algebras [LMZ];

(4) locally nilpotent derivations and cancellation problem of Artin-Schelter regular algebras [LMZ].

Several authors have been studying the Nakayama automorphism of some special classes of algebras during the last few years. Yekutieli gave an explicit formula of the Nakayama automorphism of the universal enveloping algebra $U(L)$ of a finite dimensional Lie algebra $L[$ Ye]. Rogalski-Reyes-Zhang proved several homological identities about the Nakayama automorphism in [RRZ1, RRZ2]. Liu-Wang-Wu studied the Nakayama automorphism for Ore extensions in [LWW]; in particular, they gave a description of the Nakayama automorphism of the Ore extension algebra $A[x ; \sigma, \delta]$. Zhu-Van Oystaeyen-Zhang computed the Nakayama automorphism of a trimmed double Ore extension of a Koszul Artin-Schelter regular algebra in [ZVZ]. The authors of the present paper studied group actions and Hopf algebra actions on Artin-Schelter regular algebras of global dimension three in connection with the Nakayama automorphism [LMZ]. In general, the Nakayama automorphism is a subtle invariant and difficult to compute. Therefore it is definitely worth understanding and calculating the Nakayama automorphism for more examples.

Note that the methods introduced in the papers [RRZ1, RRZ2, LWW] does not apply in our situation. We use another well-known method coming from Cassidy-Vancliff's paper [CV1], by constructing a sequence of regular normalizing elements. Consequently, the Koszul dual of $A(Q, C)$ is a complete intersection in the sense of [CV1, CV2, KKZ]. The algebras appeared in Theorem 0.2 are very easy in terms of generators and relations. It would be interesting to work out further properties concerning group or Hopf algebra actions and universal quantum linear group coactions (as in the work [WW]) on $A(Q, C)$, and invariants, such as, the center, the automorphism group, and the Makar-Limanov invariant, of $A(Q, C)$.

We finish the introduction with the following question.

Question 0.4. What is the explicit formula for the Nakayama automorphism of a graded Artin-Schelter regular skew Clifford algebra in the sense of [CV1, CV2]?

\section{ACKNOWLEDGMENTS}

J.-F. Lü was supported by NSFC (Grant Nos.11571316 and 11001245) and Natural Science Foundation of Zhejiang Province (Grant No. LY16A010003). X.-F. Mao was supported by NSFC (Grant No.11001056), the Key Disciplines of Shanghai Municipality (Grant No.S30104) and the Innovation Program of Shanghai Municipal Education Commission (Grant No.12YZ031). J.J. Zhang was supported by the US National Science Foundation (NSF grant No. DMS 1402863). 


\section{THE PROOF OF TheOREM 0.2}

In this section, we study the algebra $A(Q, C)$ defined as in the introduction. Throughout this section we assume that

\section{the parameters $(Q, C)$ satisfy conditions $(\mathrm{E} 0.0 .1)-(\mathrm{E} 0.0 .8)$.}

By definition, the algebra $A(Q, C)$ is a quadratic algebra, and its Koszul dual is

$$
A^{!}(Q, C):=\mathbb{k}\left\langle x_{1}, \cdots, x_{n}\right\rangle /(\operatorname{Rel})
$$

where $\left\{x_{1}, \cdots, x_{n}\right\}$ is the dual basis of $\left\{t_{1}, \cdots, t_{n}\right\}$ and the ideal $(\mathrm{Rel})$ is generated by the following relations.

(E1.0.1)

$$
\begin{array}{rlrl}
x_{j} x_{i}+q_{i j}^{-1} x_{i} x_{j} & =0, & & \text { for all } 1 \leq i<j \leq n, \\
x_{i}^{2} & =0, & & \text { for all } i>s, \\
x_{i}^{2}-x_{1}^{2} & =0, & & \text { for all } 1<i \leq s, \\
x_{1}^{2} & =\sum_{i<j} q_{i j}^{-1} c_{i j} x_{i} x_{j}\left(=-\sum_{i<j} c_{j i} x_{i} x_{j}\right) .
\end{array}
$$

Let $p_{i j}=-q_{i j}^{-1}$ and define

$$
T_{1}=\mathbb{k}_{p_{i j}}\left[x_{1}, \cdots, x_{n}\right]\left(:=\mathbb{R}\left\langle x_{1}, \cdots, x_{n}\right\rangle /\left(x_{j} x_{i}-p_{i j} x_{i} x_{j}, \forall i<j\right)\right) .
$$

We can construct a sequence of regular normal elements. Since $T_{1}$ is a skew polynomial ring, it is Koszul and Artin-Schelter regular of global dimension $n$. It is well-known that the monomials $\left\{x_{1}^{d_{1}} \cdots x_{n}^{d_{n}} \mid d_{s} \geq 0\right\}$ form a $\mathbb{k}$-linear basis and the Hilbert series of $T_{1}$ is $\frac{1}{(1-t)^{n}}$. Since $x_{i}^{2}$ are normal and since condition (E0.0.3), one can check, by using the monomial basis, that $\left\{x_{1}^{2}-x_{i}^{2}\right\}_{i=2}^{s} \cup\left\{x_{p}^{2}\right\}_{p=s+1}^{n}$ form a regular normal sequence of $T_{1}$. By factoring out this sequence of regular normal elements in $T_{1}$, we let

$$
T_{2}=T_{1} /\left(\left\{x_{1}^{2}-x_{i}^{2}\right\}_{i=2}^{s} \cup\left\{x_{p}^{2}\right\}_{p=s+1}^{n}\right) .
$$

Then $T_{2}$ is an Artin-Schelter Gorenstein algebra of injective dimension one and its Hilbert series is

$$
H_{T_{2}}(t)=\frac{\left(1-t^{2}\right)^{n-1}}{(1-t)^{n}}=\frac{(1+t)^{n-1}}{(1-t)} .
$$

Note that $A^{!}(Q, C)$ is a factor ring of $T_{2}$. Next we define some elements in $T_{1}$ (or $T_{2}$ or $\left.A^{!}(Q, C)\right)$. For any $1 \leq \alpha \leq n$, let

$$
\begin{gathered}
y_{\alpha}:=\sum_{i=1}^{n} c_{\alpha i} x_{i}=\sum_{i \neq \alpha} c_{\alpha i} x_{i}, \\
W_{\alpha}:=y_{\alpha} x_{\alpha}=\sum_{i<\alpha} c_{\alpha i} x_{i} x_{\alpha}+\sum_{j>\alpha} c_{j \alpha} x_{\alpha} x_{j}, \\
M_{\alpha}:=\sum_{i<j, i \neq \alpha, j \neq \alpha} c_{j i} x_{i} x_{j}, \\
\mathbb{W}:=\sum_{\alpha>s} W_{\alpha},
\end{gathered}
$$

and

$$
\phi:=x_{1}^{2}+\sum_{i<j} c_{j i} x_{i} x_{j} .
$$

Note that the algebras $T_{1}, T_{2}$ and $A^{!}(Q, C)$ and the above elements are well-defined without $(Q, C)$ satisfying (E0.0.1)-(E0.0.8), but we always assume (E0.0.1)- E0.0.8) in this section. 
The following is an easy observation.

Lemma 1.1. Parts $(1,2)$ hold in the algebra $T_{1}$ and part (3) holds in $T_{2}$.

(1) $x_{\alpha} y_{\alpha}=-y_{\alpha} x_{\alpha}$ and $x_{\alpha} W_{\alpha}=-W_{\alpha} x_{\alpha}$ for all $\alpha \leq s$.

(2) $x_{\alpha} M_{\alpha}=q_{1 \alpha}^{-2} M_{\alpha} x_{\alpha}$ for all $\alpha$, and $x_{\alpha} M_{\alpha}=M_{\alpha} x_{\alpha}$ for all $\alpha \leq s$.

(3) $W_{i} x_{i}=x_{i} W_{i}=0$ for all $i>s$.

Proof. Each follows from a direct computation under the conditions (E0.0.1)- E0.0.5).

Lemma 1.2. The following hold in $T_{2}$.

(1) $\sum_{j} c_{\alpha j} x_{j} M_{j}=\sum_{j} c_{\alpha j} M_{j} x_{j}=0$ for all $\alpha \leq s$.

(2) If $i>s$, then $x_{i} \phi=q_{1 i}^{-2} \phi x_{i}$.

(3) If $i \leq s$, then $\left(x_{i}+y_{i}\right) \phi=\phi\left(x_{i}-y_{i}\right)$.

(4) $\phi$ is normal in $T_{2}$.

Proof. (1) We use E0.0.7) in the last step of the following computation

$$
\begin{aligned}
\sum_{j} c_{\alpha j} x_{j} M_{j} & =\sum_{j} c_{\alpha j} x_{j}\left(\sum_{l<k, l \neq j, k \neq j} c_{k l} x_{l} x_{k}\right) \\
& =\sum_{j, l<k, l \neq j, k \neq j} c_{\alpha j} c_{k l} x_{j} x_{k} x_{l} \\
& =\sum_{j<l<k} c_{\alpha j} c_{k l} x_{j} x_{k} x_{l}+\sum_{l<j<k} c_{\alpha j} c_{k l} x_{j} x_{k} x_{l}+\sum_{l<k<j} c_{\alpha j} c_{k l} x_{j} x_{k} x_{l} \\
& =\sum_{j<l<k} c_{\alpha j} c_{k l} x_{j} x_{k} x_{l}+\sum_{j<l<k} c_{\alpha l} c_{k j} x_{l} x_{k} x_{j}+\sum_{j<l<k} c_{\alpha k} c_{l j} x_{k} x_{l} x_{j} \\
& =\sum_{j<l<k}\left(q_{j k} q_{j l} c_{\alpha j} c_{k l}-q_{l k} c_{\alpha l} c_{k j}+c_{\alpha k} c_{l j}\right) x_{k} x_{l} x_{j} \\
& =0 .
\end{aligned}
$$

Similarly, we use E0.0.6 in the last step of the following computation

$$
\begin{aligned}
\sum_{j} c_{\alpha j} M_{j} x_{j} & =\sum_{j} c_{\alpha j}\left(\sum_{l<k, l \neq j, k \neq j} c_{k l} x_{l} x_{k}\right) x_{j} \\
& =\sum_{j, l<k, l \neq j, k \neq j} c_{\alpha j} c_{k l} x_{k} x_{l} x_{j} \\
& =\sum_{j<l<k} c_{\alpha j} c_{k l} x_{k} x_{l} x_{j}+\sum_{l<j<k} c_{\alpha j} c_{k l} x_{k} x_{l} x_{j}+\sum_{l<k<j} c_{\alpha j} c_{k l} x_{k} x_{l} x_{j} \\
& =\sum_{j<l<k} c_{\alpha j} c_{k l} x_{k} x_{l} x_{j}+\sum_{j<l<k} c_{\alpha l} c_{k j} x_{k} x_{j} x_{l}+\sum_{j<l<k} c_{\alpha k} c_{l j} x_{l} x_{j} x_{k} \\
& =\sum_{j<l<k}\left(c_{\alpha j} c_{k l}-q_{j l} c_{\alpha l} c_{k j}+q_{l k} q_{j k} c_{\alpha k} c_{l j}\right) x_{k} x_{l} x_{j}=0 .
\end{aligned}
$$

(2) Since $i>s, x_{i}^{2}=0$ and $x_{i} W_{i}=W_{i} x_{i}=0$. By Lemma1.1 2 ), $x_{i} M_{i}=q_{1 i}^{-2} M_{i} x_{i}$. Hence

$$
\begin{aligned}
x_{i} \phi & =x_{i}\left(x_{1}^{2}+W_{i}+M_{i}\right) \\
& =x_{i} x_{1}^{2}+x_{i} M_{i} \\
& =q_{1 i}^{-2} x_{1}^{2} x_{i}+q_{1 i}^{-2} M_{i} x_{i} \\
& =q_{1 i}^{-2}\left(x_{1}^{2}+W_{i}+M_{i}\right) x_{i} \\
& =q_{1 i}^{-2} \phi x_{i} .
\end{aligned}
$$


(3) Let $\alpha \leq s$. By using the fact that $x_{\alpha}^{2}=x_{1}^{2}$ and that $\sum_{i<j} c_{j i} x_{i} x_{j}=W_{l}+M_{l}$ for all $l$, and by Lemma 1.1 and part (1), we have

$$
\begin{array}{rlr}
\left(x_{\alpha}+y_{\alpha}\right) \phi & =\left(x_{\alpha}+y_{\alpha}\right)\left(x_{\alpha}^{2}+\sum_{i<j} c_{j i} x_{i} x_{j}\right) \\
& =x_{\alpha}^{3}+y_{\alpha} x_{\alpha}^{2}+x_{\alpha}\left(W_{\alpha}+M_{\alpha}\right)+\sum_{j} c_{\alpha j} x_{j}\left(W_{j}+M_{j}\right) \\
& =x_{\alpha}^{3}+W_{\alpha} x_{\alpha}+x_{\alpha} W_{\alpha}+x_{\alpha} M_{\alpha}+\sum_{j} c_{\alpha j} x_{j} W_{j} & \text { part (1) } \\
& =x_{\alpha}^{3}-x_{\alpha} W_{\alpha}+x_{\alpha} W_{\alpha}+x_{\alpha} M_{\alpha}+\sum_{j} c_{\alpha j} x_{j} W_{j} & \text { Lemma11.1 } 1) \\
& =x_{\alpha}^{3}+x_{\alpha} M_{\alpha}+\sum_{j \leq s} c_{\alpha j} x_{j} W_{j}
\end{array}
$$

Similarly,

$$
\begin{aligned}
\phi\left(x_{\alpha}-y_{\alpha}\right) & =\left(x_{\alpha}^{2}+\sum_{i<j} c_{j i} x_{i} x_{j}\right)\left(x_{\alpha}-y_{\alpha}\right) \\
& =x_{\alpha}^{3}-x_{\alpha}^{2} y_{\alpha}+\left(W_{\alpha}+M_{\alpha}\right) x_{\alpha}-\sum_{j} c_{\alpha j}\left(W_{j}+M_{j}\right) x_{j} \\
& =x_{\alpha}^{3}-W_{\alpha} x_{\alpha}+W_{\alpha} x_{\alpha}+M_{\alpha} x_{\alpha}-\sum_{j} c_{\alpha j} W_{j} x_{j}-\sum_{j} c_{\alpha j} M_{j} x_{j} \\
& =x_{\alpha}^{3}+M_{\alpha} x_{\alpha}-\sum_{j \leq s} c_{\alpha j} W_{j} x_{j} \\
& =x_{\alpha}^{3}+x_{\alpha} M_{\alpha}+\sum_{j \leq s} c_{\alpha j} x_{j} W_{j} .
\end{aligned}
$$

So we have

$$
\left(x_{\alpha}+y_{\alpha}\right) \phi=\phi\left(x_{\alpha}-y_{\alpha}\right) .
$$

(4) By Remark 0.1, $\operatorname{det}\left(I_{s \times s}-C_{s}\right) \neq 0$ if and only if $\operatorname{det}\left(I_{s \times s}+C_{s}\right) \neq 0$. By hypothesis, $\operatorname{det}\left(I_{s \times s}-C_{s}\right) \neq 0$, whence $\left\{x_{i}-y_{i}\right\}_{i=1}^{s} \cup\left\{x_{j}\right\}_{j=s+1}^{n}$ is a basis of $V:=\bigoplus_{i=1}^{n} \mathbb{k} x_{i}$. Similarly, $\operatorname{det}\left(I_{s \times s}+C_{s}\right) \neq 0$ implies that $\left\{x_{i}+y_{i}\right\}_{i=1}^{s} \bigcup\left\{x_{j}\right\}_{j=s+1}^{n}$ is a basis of $V$. In this case, $V \phi=\phi V$ by parts $(2,3)$. Therefore $A \phi=\phi A$.

It is clear that $\sum_{i<j} c_{j i} x_{i} x_{j}=W_{\alpha}+M_{\alpha}$ for every $\alpha$.

Lemma 1.3. The following hold in $A^{!}(Q, C)$.

(1) $W_{\alpha} x_{\alpha}=x_{\alpha} W_{\alpha}=0$ for all $\alpha$.

(2) $\sum_{\alpha=1}^{n} W_{\alpha}=2 \sum_{i<j} c_{j i} x_{i} x_{j}=\sum_{\alpha=1}^{s} W_{\alpha}+\mathbb{W}$.

(3) $W_{\alpha}^{2}=0$ for all $\alpha>s$ and $\mathbb{W}^{m}=0$ for all $m>n-s$.

(4) $x_{1}^{4}=-\frac{1}{2} \mathbb{W} x_{1}^{2}=-\frac{1}{2} x_{1}^{2} \mathbb{W}$ and $x_{1}^{4 m}=0$ for all $m>n-s$.

(5) If $f \in A^{!}(Q, C)$ is a homogeneous element of degree $>4(n-s) n$, then $f=0$. As a consequence, $A^{!}(Q, C)$ is finite dimensional.

(6) The algebra $A^{!}(Q, C)$ is Koszul and Frobenius of Hilbert series $(1+t)^{n}$.

Proof. (1) If $\alpha>s$, then the assertion follows from Lemma1.13). Now assume that $\alpha \leq s$. It follows from Lemma 1.1 (1) that $x_{\alpha} W_{\alpha}=-W_{\alpha} x_{\alpha}$. By using Lemma1.1 (2) and the fact 
that $p_{1 \alpha}^{2}=1$ for all $\alpha \leq s$, we have the following computation in $A^{!}(Q, C)$ :

$$
\begin{aligned}
0 & =x_{1}^{2} x_{\alpha}-x_{\alpha} x_{1}^{2} \\
& =\left(-\sum_{i<j} c_{j i} x_{i} x_{j}\right) x_{\alpha}+x_{\alpha}\left(\sum_{i<j} c_{j i} x_{i} x_{j}\right) \\
& =-\left(W_{\alpha}+M_{\alpha}\right) x_{\alpha}+x_{\alpha}\left(W_{\alpha}+M_{\alpha}\right) \\
& =-W_{\alpha} x_{\alpha}-M_{\alpha} x_{\alpha}+x_{\alpha} W_{\alpha}+x_{\alpha} M_{\alpha} \\
& =-W_{\alpha} x_{\alpha}-M_{\alpha} x_{\alpha}-W_{\alpha} x_{\alpha}+M_{\alpha} x_{\alpha} \\
& =-2 W_{\alpha} x_{\alpha} .
\end{aligned}
$$

The assertion follows since char $\mathbb{k} \neq 2$.

(2) Clear.

(3) Note that $W_{j}=x_{j} f$ for some $f \in A^{!}(Q, C)$. The assertions follow from the fact $x_{j}^{2}=0$ for all $j>s$.

(4) We compute

$$
\begin{aligned}
-2 x_{1}^{4} & =-2 x_{1}^{2} x_{1}^{2}=2\left(\sum_{i<j} c_{j i} x_{i} x_{j}\right) x_{1}^{2}=\left(\sum_{\alpha=1}^{s} W_{\alpha}+\mathbb{W}\right) x_{1}^{2} \\
& =\sum_{\alpha=1}^{s} W_{\alpha} x_{1}^{2}+\mathbb{W} x_{1}^{2}=\sum_{\alpha=1}^{s} W_{\alpha} x_{\alpha}^{2}+\mathbb{W} x_{1}^{2}=0+\mathbb{W} x_{1}^{2}=\mathbb{W} x_{1}^{2} .
\end{aligned}
$$

Similarly, $-2 x_{1}^{4}=x_{1}^{2} \mathbb{W}$. So the first assertion follows. The second assertion follows from the first one and part (3).

(5) It follows from part (4) and the relations of $A^{!}(Q, C)$ that $x_{i}^{4 m}=0$ for all $m>n-s$. So any monomial of degree $>4(n-s) n$ is zero. The assertion follows.

(6) By Lemma 1.2(4), $\phi$ is normal in $T_{2}$. By definition, $T_{2} / \phi T_{2}=T_{2} /(\phi)=A^{!}(Q, C)$, which is finite dimensional by part (5). Let $K=\left\{x \in T_{2} \mid \phi x=0\right\}$. Then $K$ is a right graded $T_{2}$-module. Since $T_{2}$ is noetherian, PI, Artin-Schelter Gorenstein of GK-dimension one, it is Cohen-Macaulay with respect to GK-dimension [SZ, Theorem 1.1]. In particular, any nonzero submodule of $T_{2}$ has GK-dimension one by the definition of GK-Cohen-Macaulay. Consider the following exact sequence

$$
0 \rightarrow K(2) \rightarrow T_{2}(2) \stackrel{l_{\phi}}{\rightarrow} T_{2} \rightarrow T_{2} /\left(\phi T_{2}\right)\left(=A^{!}(Q, C)\right) \rightarrow 0,
$$

where $K(2)$ and $T_{2}(2)$ are the degree shift by 2 of graded modules $K$ and $T_{2}$ respectively, and note that $A^{!}(Q, C)$ is finite dimensional. Since $H_{T_{2}}(t)=\frac{(1+t)^{n}}{(1-t)}, \operatorname{dim}\left(T_{2}\right)_{d}=2^{n}$ for $d>n$. Thus the above exact sequence implies that $K_{d+2}=0$ for all $d \gg 0$. By the GK-CohenMacaulay property of $T_{2}, K=0$, and hence $\phi$ is left regular. Similarly, $\phi$ is right regular. By [CV1, Corollary 2.2], $T_{2} /(\phi)$ is Koszul and Frobenius with Hilbert series $(1+t)^{n}$.

Note that part (6) is a special case of [CV1, Corollary 2.6]. We will need the following lemma in the proof of Theorem 0.2 .

Lemma 1.4. [RRZ1, Lemma 1.5] Let A be a noetherian connected graded Artin-Schelter Gorenstein algebra and let $z$ be a homogeneous regular normal elements of positive degree such that $\mu_{A}(z)=c z$ for some $c \in \mathbb{R}^{\times}:=\mathbb{k} \backslash\{0\}$. Let $\tau$ be in $\operatorname{Aut}(A)$ such that $z a=\tau(a) z$ for all $a \in A$. Then $\mu_{A /(z)}$ is equal to $\mu_{A} \circ \tau$ when restricted to $A /(z)$.

Now, we can prove Theorem 0.2 
Proof of Theorem 0.2 (1) By Lemma 1.3 6), the algebra $A^{!}(Q, C)$ is Koszul and Frobenius with Hilbert series $(1+t)^{n}$. By Koszul duality and [Sm, Theorem 4.3 and Proposition 5.10], $A(Q, C)$ is Koszul, Artin-Schelter regular of global dimension $n$ with Hilbert series $\frac{1}{(1-t)^{n}}$. Thus part (1) follows.

(2) By Lemmas 1.2 and 1.3 ,

$$
\left\{x_{i}^{2}-x_{1}^{2}\right\}_{i=2}^{s} \cup\left\{x_{p}^{2}\right\}_{p=s+1}^{n} \cup\{\phi\}
$$

is a sequence of regular normalizing elements of degree 2 in $T_{1}$. By [ST, Corollary 1.4], $A(Q, C)$ contains a sequence of regular normalizing elements of degree 2 , say $\left\{f_{1}, \cdots, f_{n}\right\}$, such that $A(Q, C) /\left(f_{1}, \cdots, f_{n}\right)$ is finite dimensional. Using [ASZ, Proposition 4.9(1)] repeatedly, $A(Q, C)$ is strongly noetherian. It is also easy to see that $A(Q, C)$ has enough normal elements in the sense of $[\overline{\mathrm{Zh}}]$. Other assertions of part (2) follow from part (1) and [Zh, Theorem 0.2].

(3) Let us work on the algebra $T_{1}$. By [LWW, Proposition 4.1] or [RRZ1, Example 5.5], the Nakayama automorphism of $T_{1}$ is determined by

$$
\mu_{T_{1}}: x_{j} \mapsto\left(\prod_{w=1}^{n} p_{w j}\right) x_{j}=(-1)^{n}\left(\prod_{w=1}^{n} q_{j w}\right) x_{j}
$$

for all $j$. Note that each $x_{i}^{2}-x_{1}^{2}$, for $i=2, \cdots, s$, is normal and

$$
\mu_{T_{1}}\left(x_{i}^{2}-x_{1}^{2}\right)=\left(\prod_{w=1}^{n} q_{1 w}\right)^{2}\left(x_{i}^{2}-x_{1}^{2}\right)
$$

by (E0.0.3). The conjugation by $x_{i}^{2}-x_{1}^{2}$ is the automorphism determined by

$$
\tau_{x_{i}^{2}-x_{1}^{2}}: x_{j} \mapsto\left(x_{i}^{2}-x_{1}^{2}\right) x_{j}\left(x_{i}^{2}-x_{1}^{2}\right)^{-1}=q_{i j}^{2} x_{j}
$$

for all $j$. Similarly, each $x_{p}^{2}$, for $p>s$, is normal and the conjugation by $x_{p}^{2}$ is the automorphism determined by

$$
\tau_{x_{p}^{2}}: x_{j} \mapsto\left(x_{p}^{2}\right) x_{j}\left(x_{p}^{2}\right)^{-1}=q_{p j}^{2} x_{j}
$$

for all $j$. (Some details of checking the hypotheses of Lemma 1.4 is straightforward and omitted.) Applying [RRZ1, Lemma 1.5] multiple times, we have that the Nakayama automorphism of $T_{2}$ is

$$
\mu_{T_{2}}=\mu_{T_{1}} \circ \prod_{i=2}^{s} \tau_{x_{i}^{2}-x_{1}^{2}} \circ \prod_{p>s} \tau_{x_{p}^{2}}
$$

or, after simplification,

$$
\mu_{T_{2}}: x_{j} \mapsto(-1)^{n} q_{j 1}^{2}\left(\prod_{w=1}^{n} q_{w j}\right) x_{j}
$$

for all $j$. By Lemma 1.2 $\phi$ is normal in $T_{2}$ and $\mu_{T_{2}}(\phi)=\left(\prod_{w=1}^{n} q_{w 1}\right)^{2} \phi$ by E0.0.4). The conjugation by $\phi$ is determined by

$$
\tau_{\phi}: x_{j} \mapsto \phi x_{j} \phi^{-1} .
$$


By Lemma 1.2 2,3), $\tau_{\phi}$ sends $x_{j}$ to $q_{1 j}^{2} x_{j}$ when $j>s$ and $x_{j}-y_{j}$ to $x_{j}+y_{j}$ when $j \leq s$. Therefore, by linear algebra,

$$
\left[I_{s \times s}-C_{s}\right]\left(\begin{array}{c}
\tau_{\phi}\left(x_{1}\right) \\
\tau_{\phi}\left(x_{2}\right) \\
\vdots \\
\tau_{\phi}\left(x_{s}\right)
\end{array}\right)=\left[I_{s \times s}+C_{s}\right]\left(\begin{array}{c}
x_{1} \\
x_{2} \\
\vdots \\
x_{s}
\end{array}\right)+\left(\begin{array}{c}
\sum_{i=s+1}^{n} c_{1 i}\left(1+q_{1 i}^{2}\right) x_{i} \\
\sum_{i=s+1}^{n} c_{2 i}\left(1+q_{1 i}^{2}\right) x_{i} \\
\vdots \\
\sum_{i=s+1}^{n} c_{s i}\left(1+q_{1 i}^{2}\right) x_{i}
\end{array}\right) .
$$

Let $\left(a_{i j}\right)_{s \times s}=\left[I_{s \times s}-C_{s}\right]^{-1}$ and $\left(b_{i j}\right)_{s \times s}=\left[I_{s \times s}-C_{s}\right]^{-1}\left[I_{s \times s}+C_{s}\right]$. Then for any $j \leq s$, we have

$$
\tau_{\phi}\left(x_{j}\right)=\sum_{l=1}^{s} b_{j l} x_{l}+\sum_{l=1}^{s} a_{j l}\left(\sum_{i=s+1}^{n} c_{l i}\left(1+q_{1 i}^{2}\right) x_{i}\right) .
$$

Hence the Nakayama automorphism of $E:=A^{!}(Q, C)$ is given by

$$
\mu_{E}: x_{j} \mapsto \begin{cases}(-1)^{n}\left(\prod_{w=1}^{n} q_{w j}\right) x_{j}, & \text { if } j>s \\ (-1)^{n} \sum_{l=1}^{s}\left[b_{j l} q_{l 1}^{2}\left(\prod_{w=1}^{n} q_{w l}\right) x_{l}+a_{j l}\left(\sum_{i=s+1}^{n} c_{l i}\left(1+q_{i 1}^{2}\right)\left(\prod_{w=1}^{n} q_{w i}\right) x_{i}\right)\right], & \text { if } j \leq s .\end{cases}
$$

By [RRZ2, Theorem 4.3(2)], we have $\left.\mu_{E}\right|_{E_{1}}=(-1)^{n}\left(\left.\mu_{A}\right|_{A_{1}}\right)^{*}$. Thus the Nakayama automorphism of $A(Q, C)$ is given by

$$
\mu_{A}: t_{j} \mapsto \begin{cases}\sum_{i=1}^{s} \sum_{l=1}^{s} a_{i l} c_{l j}\left(1+q_{j 1}^{2}\right)\left(\prod_{w=1}^{n} q_{w j}\right) t_{i}+\left(\prod_{w=1}^{n} q_{w j}\right) t_{j}, & \text { if } j>s \\ \sum_{i=1}^{s} b_{i j} q_{j 1}^{2}\left(\prod_{w=1}^{n} q_{w j}\right) t_{i}, & \text { if } j \leq s .\end{cases}
$$

Graded skew Clifford algebras were introduced and studied by Cassidy-Vancliff in [CV1, CV2]. Let $\mu:=\left(\mu_{i j}\right) \in M_{n}(\mathbb{k})$ such that $\mu_{i i}=1$ and $\mu_{i j} \mu_{j i}=1$ for all $1 \leq i, j \leq n$. A matrix $M:=\left(M_{i j}\right) \in M_{n}(\mathbb{k})$ is called $\mu$-symmetric if $M_{i j}=\mu_{i j} M_{j i}$ for all $i, j$, see [CV1, Definition 1.2]. Suppose that $M_{1}, \cdots, M_{n}$ are all $\mu$-symmetric $n \times n$-matrices. Following [CV1, Definition 1.12], a graded skew Clifford algebra associated to $\mu$ and $M_{1}, \cdots, M_{n}$ is a graded $\mathbb{k}$-algebra $A$ on degree-one generators $x_{1}, x_{2}, \cdots, x_{n}$ and on degree-two generators $y_{1}, y_{2}, \cdots, y_{n}$ with defining relations given by the following:

- $x_{i} x_{j}+\mu_{i j} x_{j} x_{i}=\sum_{l=1}^{n}\left(M_{l}\right)_{i j} y_{l}$ for all $i, j=1,2, \cdots, n$; and

- the existence of a normalizing sequence $\left\{r_{1}, \cdots, r_{n}\right\}$ of $A$ that spans $\mathbb{k} y_{1}+\cdots+\mathbb{k} y_{n}$.

Cassidy-Vancliff [CV1, CV2] associated the geometric data to a graded skew Clifford algebra by using noncommutative algebraic geometry developed in [ATV1, ATV2]. They generalized the notion of a quadric and a quadric system in commutative algebra to the noncommutative case, and proved that a graded skew Clifford algebra is Artin-Schelter regular if and only if its associated quadratic system is normalizing and base point free.

Proposition 1.5. Let $\Omega:=\sum_{i=1}^{s} t_{i}^{2}$. Then $\Omega$ is a regular normal element of $A:=A(Q, C)$ and $A$ is a graded skew Clifford algebra. 
Proof. Let $B=\mathbb{K}_{q_{i j}}\left[t_{1}, \cdots, t_{n}\right]$ and also consider $\Omega$ as an element in $B$. By (E0.0.3), $t_{j} \Omega=$ $q_{1 j}^{2} \Omega t_{j}$ for all $j$. Hence, $\Omega$ is a regular normal element in $B$, and the Hilbert series of $B /(\Omega)$ is $\frac{\left(1-t^{2}\right)}{(1-t)^{n}}$. By comparing the relations, one sees that

$$
A /(\Omega) \cong B /(\Omega) .
$$

Hence we have a surjective map

$$
A / \Omega A \stackrel{f}{\rightarrow} A /(\Omega) \cong B /(\Omega) .
$$

By Theorem 0.2 (2), $A$ is a domain. Then $\Omega \in A$ is regular and the Hilbert series of $A / \Omega A$ is $\frac{\left(1-t^{2}\right)}{(1-t)^{n}}$, which equals the Hilbert series of $B /(\Omega)$. This implies that $\Omega A=(\Omega) \subseteq A$. By symmetry, $A \Omega=(\Omega)$. Therefore $\Omega$ is a normal element in $A$.

Now let $\Omega_{1}=\Omega$ and $\Omega_{i}=t_{i}^{2}$ for $i=2, \cdots, n$. After we showed that $\Omega_{1}$ is a normal element in $A$, one sees that $\left\{\Omega_{1}, \Omega_{2}, \cdots, \Omega_{n}\right\}$ is a normalizing sequence of $A$, which plays the role of $\left\{r_{1}, \cdots, r_{n}\right\}$ in [CV1, Definition 1.12]. Then $t_{i}$ s play the role of $x_{i}$ s and $t_{i}^{2}$ s play the role of $y_{i}$ in [CV1, Definition 1.12]. Let $\mu$ be $Q$. Then one can easily recover the set of matrices $M_{1}, \cdots, M_{n}$ by the relations of $A$. Therefore $A$ is a graded skew Clifford algebra by [CV1, Definition 1.12].

Proposition 1.6. Let $s<n$. Let $Q_{s}:=\left(q_{i j}\right)_{s \times s}$ and $C_{s}:=\left(c_{i j}\right)_{s \times s}$ and let $A\left(Q_{s}, C_{s}\right)$ be the subalgebra of $A(Q, C)$ generated by $\left\{t_{1}, \cdots, t_{s}\right\}$. Then $A\left(Q_{s}, C_{s}\right)$ is Artin-Schelter regular and $A(Q, C)$ is an iterated Ore extension of $A\left(Q_{s}, C_{s}\right)$.

Proof. Note that E0.0.1)-(E0.0.8 hold for the submatrices $\left(Q_{s}, C_{s}\right)$. The first assertion follows from Theorem 0.2 . For each $j>s$, using the relations

$$
t_{j} t_{i}=q_{i j} t_{i} t_{j}+c_{i j}\left(\sum_{i=1}^{s} t_{s}^{2}\right), \quad \forall i<j,
$$

one can easily check that $A(Q, C)$ is an iterated Ore extension of $A\left(Q_{s}, C_{s}\right)$ by adding $t_{s+1}, t_{s+2}, \cdots, t_{n}$ consecutively.

It is possible that $A(Q, C)$ in Theorem 0.2 is always an iterated Ore extension from $\mathbb{k}$, which can be verified for all examples in the next section.

\section{EXAMPLES}

In this section we use the main result to construct some Artin-Schelter regular algebras of low global dimension and to calculate their Nakayama automorphisms.

Example 2.1. Let $n=3, s=1$ and $Q=\left(\begin{array}{ccc}1 & 1 & 1 \\ 1 & 1 & q \\ 1 & q^{-1} & 1\end{array}\right)$ and $C=\left(\begin{array}{ccc}0 & 0 & 0 \\ 0 & 0 & 1 \\ 0 & -q^{-1} & 0\end{array}\right)$. All conditions (E0.0.1)-(E0.0.8) are satisfied. The algebra is isomorphic to

$$
\mathbb{k}\left\langle t_{1}, t_{2}, t_{3}\right\rangle\left(t_{3} t_{2}-q t_{2} t_{3}-t_{1}^{2}, t_{1} \text { central }\right) .
$$

This is an Artin-Schelter regular of dimension 3, which is the algebra $A(2)$ in [LMZ]. The Nakayama automorphism of $A(Q, C)$ is given by

$$
\mu_{A}: t_{1} \mapsto t_{1}, \quad t_{2} \mapsto q^{-1} t_{2}, \quad t_{3} \mapsto q t_{3} .
$$

When $q$ is not a root of unity, the group actions, Hopf algebra actions and cancellation property of this algebra was studied in [LMZ]. 
When $n=4$, there are many solutions to system (E0.0.1)- $(\mathrm{E} 0.0 .8)$ (for example, solutions can be listed by Maple). We pick two of them and use Theorem 0.2 to calculate their Nakayama automorphism. It is possible that these algebras are isomorphic, in a nonobvious way, to some algebras constructed and studied in [CV1, CV2, NV, VV].

Example 2.2. Let $n=4, s=2, Q=\left(\begin{array}{cccc}1 & -1 & \frac{1}{b} & -1 \\ -1 & 1 & \frac{1}{b} & 1 \\ b & b & 1 & b \\ -1 & 1 & \frac{1}{b} & 1\end{array}\right)$ and $C=\left(\begin{array}{cccc}0 & 0 & 0 & 0 \\ 0 & 0 & 0 & a \\ 0 & 0 & 0 & 0 \\ 0 & -a & 0 & 0\end{array}\right)$.

Then

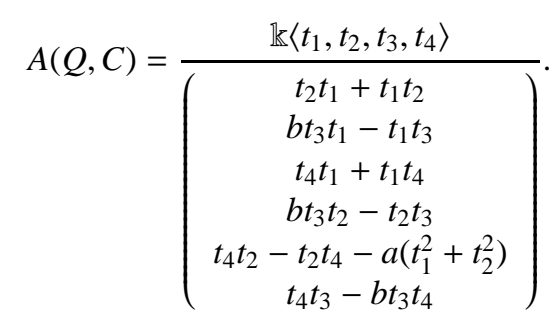

We have

$$
\left(I_{2 \times 2}-C_{2}\right)=\left(\begin{array}{cc}
1 & 0 \\
0 & 1
\end{array}\right)=I_{2 \times 2}, \quad\left(I_{2 \times 2}-C_{2}\right)^{-1}=I_{2 \times 2}
$$

and

$$
\left(I_{2 \times 2}-C_{2}\right)^{-1} \cdot\left(I_{2 \times 2}+C_{2}\right)=I_{2 \times 2} .
$$

By Theorem 0.2 (3), the Nakayama automorphism is given by

$$
\mu_{A}: \quad t_{1} \mapsto b t_{1}, \quad t_{2} \mapsto-b t_{2}, \quad t_{3} \mapsto \frac{1}{b^{3}} t_{3}, \quad t_{4} \mapsto-2 a b t_{2}-b t_{4} .
$$

Example 2.3. Let $n=4, s=3$ and $Q=\left(\begin{array}{cccc}1 & -1 & 1 & \frac{1}{b} \\ -1 & 1 & -1 & \frac{1}{b} \\ 1 & -1 & 1 & \frac{1}{b} \\ b & b & b & 1\end{array}\right)$ and $C=\left(\begin{array}{cccc}0 & 0 & a & 0 \\ 0 & 0 & 0 & 0 \\ -a & 0 & 0 & 0 \\ 0 & 0 & 0 & 0\end{array}\right)$ with $1+a^{2} \neq 0, a \in \mathbb{k}$ and $b \in \mathbb{R}^{\times}$. Then

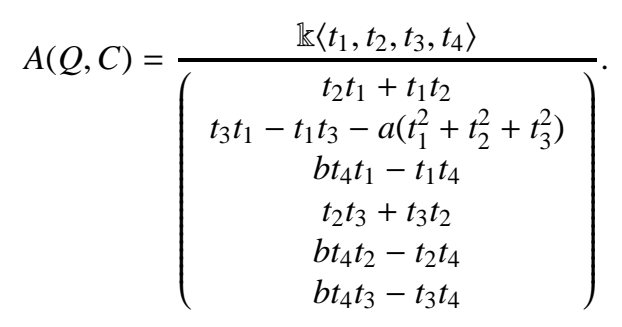

We have

$$
\left(I_{3 \times 3}-C_{3}\right)=\left(\begin{array}{ccc}
1 & 0 & -a \\
0 & 1 & 0 \\
a & 0 & 1
\end{array}\right), \quad\left(I_{3 \times 3}-C_{3}\right)^{-1}=\left(\begin{array}{ccc}
\frac{1}{1+a^{2}} & 0 & \frac{a}{1+a^{2}} \\
0 & 1 & 0 \\
\frac{-a}{1+a^{2}} & 0 & \frac{1}{1+a^{2}}
\end{array}\right)
$$

and

$$
\left(I_{3 \times 3}-C_{3}\right)^{-1} \cdot\left(I_{3 \times 3}+C_{3}\right)=\left(\begin{array}{ccc}
\frac{1-a^{2}}{1+a^{2}} & 0 & \frac{2 a}{1+a^{2}} \\
0 & 1 & 0 \\
\frac{-2 a}{1+a^{2}} & 0 & \frac{1-a^{2}}{1+a^{2}}
\end{array}\right)
$$


By Theorem 0.2 its Nakayama automorphism is given by

$$
\mu_{A}: t_{j} \mapsto \begin{cases}\frac{a^{2} b-b}{1+a^{2}} t_{1}+\frac{2 a b}{1+a^{2}} t_{3}, & \text { if } j=1 \\ b t_{2}, & \text { if } j=2 \\ \frac{-2 a b}{1+a^{2}} t_{1}+\frac{a^{2} b-b}{1+a^{2}} t_{3}, & \text { if } j=3 \\ \frac{1}{b^{3}} t_{4}, & \text { if } j=4 .\end{cases}
$$

Example 2.4. Let $s=n, q_{i j}=1$ for all $i, j$, and $C$ be a skew symmetric matrix. Then (E0.0.1)-(E0.0.5) are trivially satisfied. Equation $(\mathrm{E} 0.0 .8)$ is saying that

$$
\operatorname{det}\left(I_{n \times n}-C\right) \neq 0 .
$$

It is not hard to check that (E0.0.6) is equivalent to E0.0.7), and equivalent to

$$
c_{i j} c_{k l}+c_{j k} c_{i l}-c_{i k} c_{j l}=0, \quad \forall 1 \leq i<j<k<l \leq n .
$$

Let $A(Q, C)$ be the algebra defined by

$$
A(Q, C)=\mathbb{K}\left\langle t_{1}, \cdots, t_{n}\right\rangle /\left(t_{j} t_{i}-t_{i} t_{j}-c_{i j}\left(\sum_{l=1}^{n} t_{l}^{2}\right), \forall i<j\right) .
$$

When $C$ satisfies (E2.4.1) and (E2.4.2), by Theorem $0.2, A(Q, C)$ is a Koszul, strongly noetherian, Auslander regular algebra of global dimension $n$ and its Nakayama automorphism is determined by

$$
\mu_{A}: t_{j} \mapsto \sum_{i=1}^{n} b_{i j} t_{i}, j=1,2, \cdots, n,
$$

where $\left(b_{i j}\right)_{n \times n}=\left(I_{n \times n}-C\right)^{-1}\left(I_{n \times n}+C\right)$.

It is clear that $\left(b_{i j}\right)_{n \times n}$ is the identity matrix if and only if $C$ is zero. Therefore $A(Q, C)$ is Calabi-Yau if and only if $C=0$ if and only if $A(Q, C)=\mathbb{k}\left[t_{1}, \cdots, t_{n}\right]$.

Note that the algebra $A(Q, C)$ is a deformation quantization of the Poisson algebra given in [LWZ, Section 3.2]. By [LWZ, Lemma 3.2], (E2.4.2) is equivalent to rank $C \leq 2$. When $\mathbb{k}$ is algebraically closed, it follows from [DRZ, Theorems 2.1 and 2.5] that every skew symmetric matrix $C$ with rank at most $2($ when $n=4)$ is orthogonally similar to either

$$
\left(\begin{array}{cccc}
0 & a & 0 & 0 \\
-a & 0 & 0 & 0 \\
0 & 0 & 0 & 0 \\
0 & 0 & 0 & 0
\end{array}\right) \text {, or }\left(\begin{array}{cccc}
0 & i & 0 & 0 \\
-i & 0 & 1 & 0 \\
0 & -1 & 0 & 0 \\
0 & 0 & 0 & 0
\end{array}\right) \text {, or } \quad\left(\begin{array}{cccc}
0 & -1 & i & 0 \\
1 & 0 & 0 & -i \\
-i & 0 & 0 & -1 \\
0 & i & 1 & 0
\end{array}\right)
$$

where $a \in \mathbb{K}$ and $i^{2}=-1$. This implies that there are only three isomorphism classes of $A(Q, C)$ corresponding to the above three matrices respectively (the assertion is also valid when $n>4)$. In all cases, one can show that $A(Q, C)$ is an iterated Ore extension from $\mathbb{k}$ (details are omitted).

\section{REFERENCES}

[ASZ] M. Artin, L.W. Small and J.J. Zhang, Generic flatness for strongly noetherian algebras, J. Algebra 221 (1999), no. 2, 579-610.

[ATV1] M. Artin, J.Tate and M. Van den Bergh, Some algebras associated to automorphisms of elliptic curves, The Grothendieck Festschrift, Vol. 1 (eds P. Cartier et al.; Birkhäuser, Boston, 1990) 33-85.

[ATV2] M. Artin, J. Tate and M. Van den Bergh, Modules over regular algebras of dimension 3, Invent. Math. 106 (1991), no. 2, 335-388.

[CV1] T. Cassidy and M. Vancliff, Generalizations of Graded Clifford Algebras and of Complete Intersections, J. Lond. Math. Soc. 81 (2010), 91-112.

[CV2] T. Cassidy and M. Vancliff, Corrigendum to "Generalizations of Graded Clifford Algebras and of Complete Intersections", J. Lond. Math. Soc. (2) 90 (2014), no. 2, 631-636. 
[DRZ] D.Ž. Doković, K. Rietsch and K.-M. Zhao, Normal forms for orthogonal similarity classes of skewsymmetric matrices, J. Algebra 308 (2007), 686-703.

[KKZ] E. Kirkman, J. Kuzmanovich and J.J. Zhang, Noncommutative complete intersections, J. Algebra 429 (2015), 253-286

[Le] T. Levasseur, Some properties of non-commutative regular graded rings, Glasg. Math. J. 34 (1992), 227300.

[LWW] L.-Y. Liu, S.-Q. Wang and Q.-S. Wu, Twisted Calabi-Yau property of Ore extensions, J. Noncommut. Geom. 8 (2014), no. 2, 587-609.

[LMZ] J.-F. Lü, X.-F. Mao and J.J. Zhang, Nakayama automorphism and applications, Trans. Amer. Math. Soc., (to appear), preprint arXiv: 1408.5761

[LWZ] J.-F. Lü, X. Wang and G. Zhuang, Universal enveloping algebras of Poisson Ore extensions, Proc. Amer. Math. Soc. 143 (2015), no. 11, 4633-4645.

[NV] M. Nafari and M. Vancliff, Graded skew Clifford algebras that are twists of graded Clifford algebras, Comm. Algebra 43 (2015), no. 2, 719-725.

[RRZ1] M. Reyes, D. Rogalski and J.J. Zhang, Skew Calabi-Yau algebras and homological identities, Adv. Math. 264 (2014), 308-354.

[RRZ2] M. Reyes, D. Rogalski and J.J. Zhang, Skew Calabi-Yau triangulated categories and Frobenius Extalgebras, Trans. Amer. Math. Soc., (to appear), preprint (2014) arXiv:1408.0536

[ST] B. Shelton and C. Tingey, On Koszul algebras and a new construction of ArtinSchelter regular algebras, $J$. Algebra 241 (2001), 789-798.

[Sm] S.P. Smith, Some finite-dimensional algebras related to elliptic curves, Representation theory of algebras and related topics, (Mexico City, 1994), 315-348, CMS Conf. Proc., 19. Amer. Math. Soc., Providence, RI, 1996.

[SZ] J.T. Stafford and J.J. Zhang, Homological properties of (graded) noetherian PI rings, J. Algebra 168 (1994), 998-1026

[V1] M. Van den Bergh, Existence theorems for dualizing complexes over non-commutative graded and filtered rings, J. Algebra 195 (2) (1997), 662-679.

[V2] M. Van den Bergh, A relation between Hochschild homology and cohomology for Gorenstein rings, Proc. Amer. Math. Soc. 126 (5) (1998), 1345-1348; M. Van den Bergh, Proc. Amer. Math. Soc. 130 (9) (2002), 2809-2810.

[VV] M. Vancliff and P.P. Veerapen, Point modules over regular graded skew Clifford algebras, J. Algebra 420 (2014), 54-64.

[WW] C. Walton and X. Wang, On quantum groups associated to non-Noetherian regular algebras of dimension 2, preprint (2015), arXiv: 1503.09185

[Ye] A. Yekutieli, The rigid dualizing complex of a universal enveloping algebra, J. Pure Appl. Algebra 150 (2000), no. 1, 85-93.

[Zh] J.J. Zhang, Connected graded Gorenstein algebras with enough normal elements, J. Algebra 189 (1997), no. $2,390-405$.

[ZZ] J.J. Zhang and J. Zhang, Double extension regular algebras of Type (14641), J. Algebra 322 (2009), no.2, 373-409.

[ZVZ] C. Zhu, F. Van Oystaeyen and Y.H. Zhang, Calabi-Yau extension and localization of Koszul regular algebras, preprint (2014), arXiv:1401.0330

(Lü) Department of Mathematics, Zhejiang Normal University, Jinhua 321004, China

E-mail address: jiafenglv@zjnu.edu.cn

(Mao) Department of Mathematics, Shanghai University, Shanghai 200444, China

E-mail address: xuefengmao@shu.edu.cn

(Zhang) Department of Mathematics, University of Washington, Seattle, WA 98195, USA

E-mail address: zhang@washington.edu 